\title{
Studies on Red-Shift Rules in Fluorescence Spectra of Human Blood Induced by LED
}

\author{
Changde Peng ${ }^{1,2} \&$ Jinsong Liu ${ }^{1}$ \\ ${ }^{1}$ College of Optoelectronic Science and Engineering, Huazhong University of Science and Technology, Wuhan, \\ China \\ ${ }^{2}$ College of Physics and Electronic Engineering, Jiangsu Normal University, Xuzhou, China \\ Correspondence: Changde Peng, College of Physics and Electronic Engineering, Jiangsu Normal University, \\ Xuzhou 221116, China. Tel: 86-13852470080. E-mail: pengchangde@126.com
}

Received: August 21, 2012 Accepted: September 10, 2012 Online Published: January 5, 2013

doi:10.5539/apr.v5n1p1 URL: http://dx.doi.org/10.5539/apr.v5n1p1

\begin{abstract}
The characteristics of fluorescent spectrum of human blood induced by LED light have been studied in this paper. The results show that the fluorescent peak appears natural exponentially shift toward longer wavelength with the increment of blood concentration. The mechanism of energy absorption between fluorophores under different blood concentrations has been analyzed. Moreover, energy transformation between different fluorophores under the same blood concentration but different fluorescent areas has been studied. The study reveals that absorption is the main reason of red-shift.
\end{abstract}

Keywords: fluorescence spectra, human blood, energy transformation, red-shift

\section{Introduction}

Recently, the laser induced auto-fluorescence (LIAF) diagnostics is extensively used in diagnosing and measuring diseases such as early-cancer and so on (Al-Salhi, Masilamani, Vijmasi, Al-Nachawati, \& VijayaRaghavan, 2011; de Oliveira Silva et al., 2010; Kalnina et al., 2010; Masilamani et al., 2011). However, the current diagnostic method is to measure the fluorescent spectra of the normal and diseased tissue samples and find out the structural difference between them (de Goes Rocha et al., 2010; Fauaz et al., 2010; Karadaglic, Wood, McRobbie, Stojanovic, \& Herrington, 2009). Since human bodies are complexity that even if the same healthy tissue can emit different fluorescence spectra under the different environment. Therefore fluorescence spectra cannot truly reflect the structure characteristic of endogenous fluorescence spectra about the flurorophores inside tissue. The deeper investigation on structure characteristic and the changes of fluorescence spectra may offer a theoretical reference to correctly differentiate the fluorescence spectra of the normal and pathological tissue.

The biomolecular have photosensitive materials inside. Without adding additional photosensitive materials such as hematoporphyrin, biological tissue may emit auto-fluorescence excited by the light. Therefore, the autofluorescence is the intrinsic reflection of biological tissue. The bilirubin, riboflavin, porphyrin, $\mathrm{VB}_{6}$ are endogenous fluorescent materials inside biological organization. The amount of fluorescenct material in biological organization may directly affect the structure of fluorescence spectra. As for fluorescenct material in pathological and normal tissue has different content and would emit different fluorescence spectra, it can distinguish pathological tissue from normal one. As a result, the investigation on characteristic of fluorescence spectra stimulated by different content of fluorescence material will make sense in future.

To enhance the monitor sensitivity and diagnostic result, some affinity fluorescence stain such as HpD will be chosen beforehand. Although it has satisfied results of tissue diagnosis by using the fluorescence stain, the additional stain has effect on the physiological and pathological situation, and make out some bad-side effect on physiology ability of normal cells. Based on the intrinsic characteristic of fluorescence spectra, the LIAF diagnosis is totally non-attacked diagnostic skill. Laser has strong selectiveness to the flurorophores because of its narrow width; this makes the intensity of fluorescence too weak. As explored investigation, the light emitting diode (LED) is chose for the exciting light source. The pulse width of LED is about ten nanometers, and can be seen as monochromatic illuminate. It can make more flurorophore in blood emitting fluorescence, and get 
stronger fluorescence spectra. To enhance the fluorescence efficiency, the center wavelength is chose at $407 \mathrm{~nm}$. According to our results of fluorescence spectra, the fluorescence spectra of human blood stimulated by LED with wavelength of $407 \mathrm{~nm}$ show vast intensity and obvious characteristic structure (Gao et al., 2002; Peng et al., 2003).

\section{Experiments and Results}

\subsection{Experimental Equipment}

A Shimadzo UV-2201 UV-Vis spectrophotometer (Shimadzu Corporation, Kyoto, Japan) was used to measure absorption spectra of blood samples. A WGD-8 grating spectrometer (1200/mm, Gangdong Corp., Tianjing, China) was used to measure the fluorescence spectra. A photomultiplier (PMT) R955 (Huamamatsu Poton techniques Inc., Japan) was used to measure fluorescence intensity and the PMT output was directly sent to computer for analysis. Excitation sources was LED light with wavelength at $407 \mathrm{~nm}, 475 \mathrm{~nm}, 508 \mathrm{~nm}$ and 570 $\mathrm{nm}$, with $5 \mathrm{~mW}$ power and $18 \mathrm{~nm}$ pulse width $\left(\Delta \lambda_{1 / 2}\right)$.

\subsection{Experimental Samples}

The whole blood solution was mixed with fresh blood taking from a 30 years old healthy man and 5\% heparin as anticoagulant. The whole blood was diluted into appropriate concentration by physiological saline water.

\subsection{Experimental Method}

$2 \mathrm{ml}$ whole blood sample was put into a quartz cuvette (QC), and the same amount of physiological saline water is put into another QC as a control sample. Then, the absorption spectra of the whole blood solution from 350 to $800 \mathrm{~nm}$ were scanned by the UV-2201 spectrophotometer, and the parameter of scan step is $0.1 \mathrm{~nm}$. The whole experiments are made at room temperature.

\subsection{Results}

The fluorescence spectra and absorption spectra of $0.8 \%$ whole blood solution from $350 \mathrm{~nm}$ to $800 \mathrm{~nm}$ are shown in Figure 1. The curve 1 shows the fluorescence spectra excited by LED light at $407 \mathrm{~nm}$, and curve 2 shows the absorption spectra.

The fluorescence spectra of the whole blood solution from $570 \mathrm{~nm}$ to $800 \mathrm{~nm}$ with different concentration excited by LED light at $407 \mathrm{~nm}$ are shown in Figure 2.

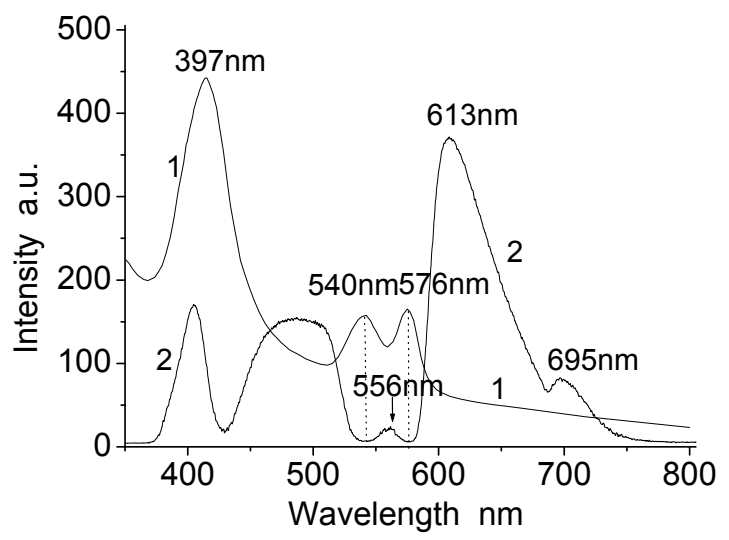

Figure 1. The fluorescence spectra and absorption spectra of human whole blood solution 


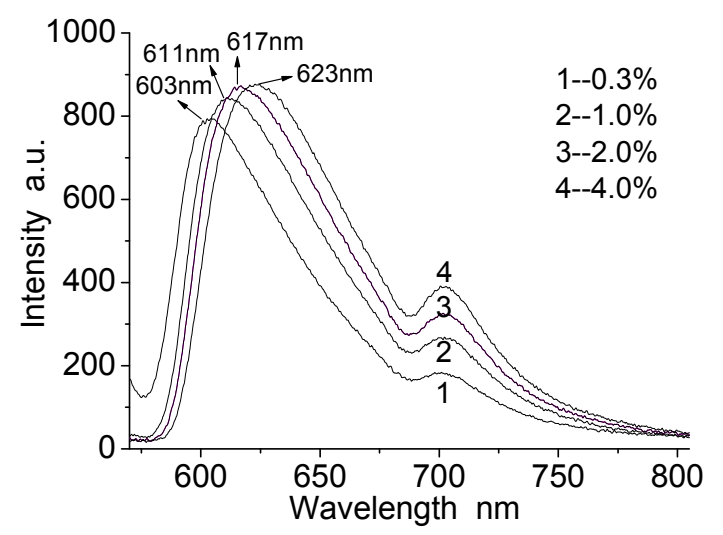

Figure 2. Red-shifted phenomenon of the whole blood solution with different concentrations

\section{Discussions}

It can be seen from Figure 2 that when the whole blood with different concentrations excited by the same light, fluorescence spectral peaks from $570 \mathrm{~nm}$ to $800 \mathrm{~nm}$ are apparently shifting to longer wavelength with the increment of blood concentration, i.e. the red-shifted. Using the curve fitting, it can be seen that the peak is red-shifted in compliance with the rule of e-exponent (Figure 3 and Figure 4). The curve formula is

$$
y=y_{0}+A_{1} \exp \frac{\left(x-x_{0}\right)}{t_{1}}
$$

The experimental results indicate that after altering the wavelength of excitation light, the fluorescence peak is still red shifted in compliance with the rule of e-exponent with the increase of the blood concentration, and only the formula parameters change. Figure 3 and Figure 4 show the shift rule of the fluorescence spectra peak with the concentration from $0.3 \%$ to $15 \%$ excited by LED light at $407 \mathrm{~nm}$ and $570 \mathrm{~nm}$, respectively. The two figures are both in compliance with e-exponent while the parameters are different.

Figure 5 shows the relationship between the fluorescence spectra peaks and the concentration of blood from $0.3 \%-50 \%$ excited by LED light at $407 \mathrm{~nm}$. The result indicates that when the concentration reaches $15 \%$, the phenomenon of fluorescence peak red-shifted following with the increase of the blood concentration disappeared. Let $C_{e}$ to express this concentration. The further experimental results show $C_{e}$ will change when the excitation light alter. The shorter wavelength the excited LED light has, the smaller $C_{e}$ is; and vice versa. Figure 3 and Figure 4 have showed this characteristic, too.

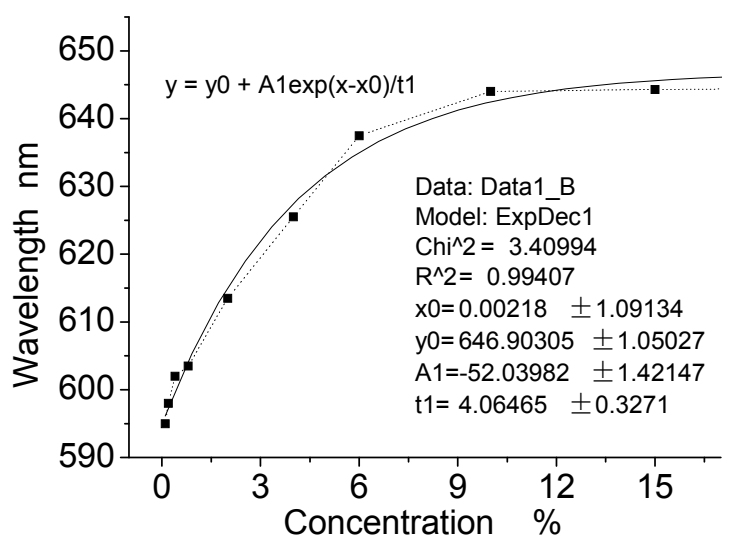

Figure 3. Fluorescence peak shifts excited by LED light at $407 \mathrm{~nm}$ 


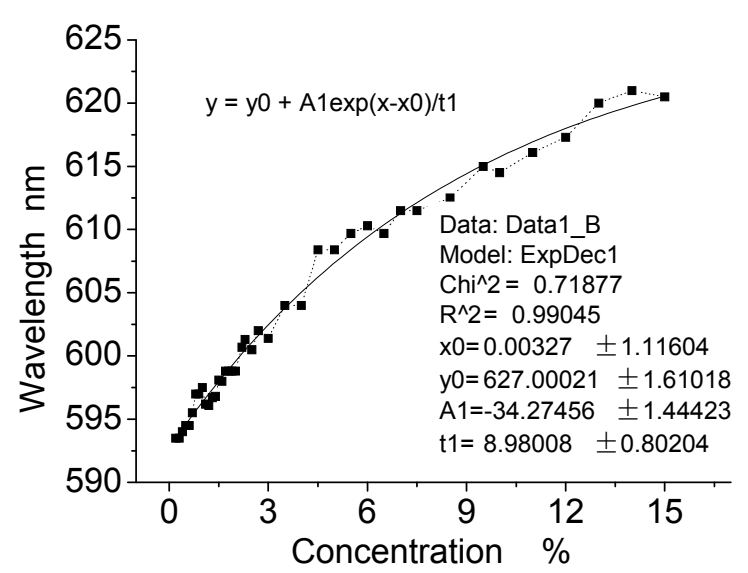

Figure 4. Fluorescence peak shifts excited by LED light at 570nm

The phenomenon that fluorescence spectral peaks red-shifted with the increase of blood concentration can be explained by the knowledge of blood absorption. It can be seen from Figure 1 that the absorption spectra (curve 1) has stronger absorptivity in short-wave area, especially at wavelength of $417 \mathrm{~nm}$; while in long-wave area, the absorptivity is lower, and especially when the wavelength is longer than $600 \mathrm{~nm}$. The absorption spectra and fluorescence spectra are overlapped in most extension. Since there has various fluorophores in human blood and the energy transfer between fluorophores cannot be avoided, some of the fluorescence will be absorbed by the ground state molecules, and that is the radiant energy transfer process from excited molecules to the same matter of ground state ones. With blood concentration increasing, the self-absorption phenomenon increases. The lower of blood concentration, the weaker of absorption, and the fluorescence peak is lean to short wave; the higher of blood concentration, the stronger of absorption, and the fluorescence peak is lean to long wave. The absorption reaches saturation when the blood concentration achieves or exceeds the $C_{e}$, and the fluorescence peak stops shifting to the larger wavelength.

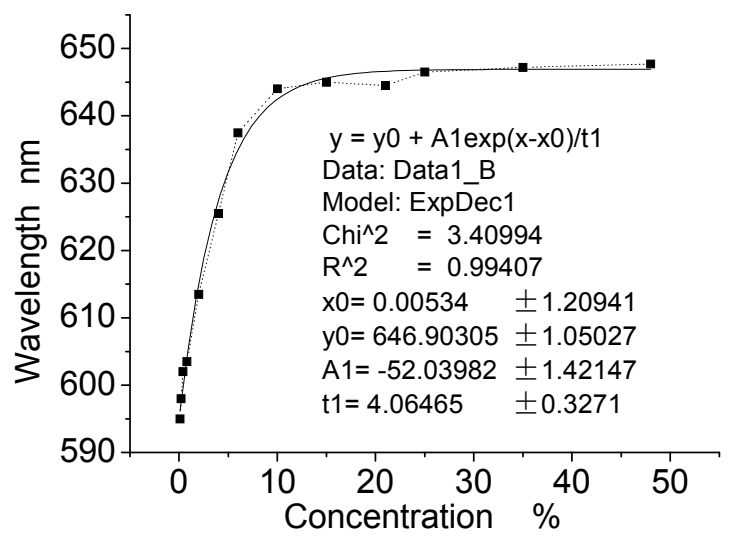

Figure 5. The red shifted phenomenon disappears when the solution concentration is more than $10 \%$ 


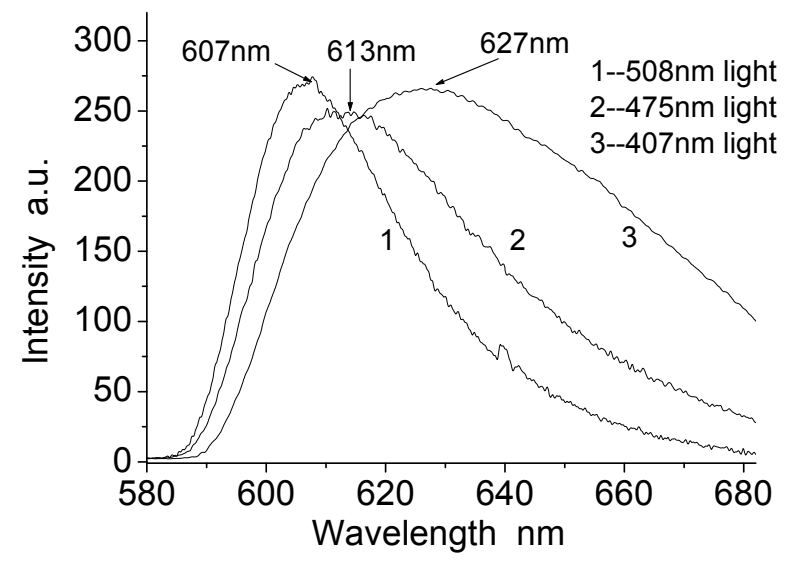

Figure 6. The fluorescence spectra of blood solution with the concentration of 3\% excited by different LED light

Figure 6 shows that the fluorescence spectral peak of the whole blood with the concentration of $3 \%$ is different when the wavelength of excited LED light is $407 \mathrm{~nm}, 475 \mathrm{~nm}$ and $508 \mathrm{~nm}$. The shorter of the excited wavelength, the longer wavelength of the peak is; and vice versa. The reason for this phenomenon is that when the wavelength of excited light is short, the fluorescence of blood will be reabsorbed and forms second-order fluorescence. And that will enhance the fluorescence efficiency, which is equivalent to enhance the fluorescence efficiency by increasing the blood concentration. That means the fluorescence peak is red-shifted with the increase of blood concentration when the excited light fixed; and it also is red-shifted with the decrease the wavelength of excited light when the blood concentration fixed. This further approves that the primary reason for red-shifted is absorption.

The results mentioned above may help us to judge the content and aberrance of fluorescent material in blood by the change of blood fluorescence peaks positions. And give reference to the differentiation and analyze of normal and pathological organic tissue fluorescence spectra in LIAF diagnostic techniques.

\section{References}

Al-Salhi, M., Masilamani, V., Vijmasi, T., Al-Nachawati, H., \& Vijaya Raghavan, A. P. (2011). Lung cancer detection by native fluorescence spectra of body fluids-a preliminary study. Journal of fluorescence, 21(2), 637-645. http://dx.doi.org/10.1007/s10895-010-0751-9

De Goes Rocha, F. G., Barbosa Chaves, K. C., Gomes, C. Z., Campanharo, C. B., Courrol, L. C., Schor, N., \& Bellini, M. H. (2010). Erythrocyte Protoporphyrin Fluorescence as a Biomarker for Monitoring Antiangiogenic Cancer Therapy. Journal of fluorescence, 20(6), 1225-1231. http://dx.doi.org/10.1007/s10895-010-0672-7

De Oliveira Silva, F. R., Bellini, M. H., Tristao, V. R., Schor, N., Vieira, N. D. Jr., \& Courrol, L. C. (2010). Intrinsic fluorescence of protoporphyrin IX from blood samples can yield information on the growth of prostate tumours. Journal of fluorescence, 20(6), 1159-1165. http://dx.doi.org/10.1007/s10895-010-0662-9

Fauaz, G., Miranda, A. R., Gomes, C. Z., Courrol, L. C., de Oliveira Silva, F. R., de Goes Rocha, F. C., ... Bellini, M. H. (2010). Erythrocyte Protoporphyrin Fluorescence as a Potential Marker of Diabetes. Applied Spectroscopy, 64(4), 391-395. http://dx.doi.org/10.1366/000370210791114248

Gao, S., Luo, X., Lan, X., Liu, Y., Lu, J., \& Ni, X. (2002). Analyzing of LED-induced blood fluorescent spectra. Chinese journal of lasers, 11(2), 315-318.

Kalnina, I., Kurjane, N., Kirilova, E., Klimkane, L., Kirilov, G., \& Zvagule, T. (2010). Correlation of altered blood albumin characteristics and lymphocyte populations to tumor stage in gastrointestinal cancer patients. Cancer biomarkers : section A of Disease markers, 7(2), 91-99. http://dx.doi.org/10.3233/CBM-2010-0151

Karadaglic, D., Wood, A. D., McRobbie, M., Stojanovic, R., \& Herrington, C. S. (2009). Fluorescence spectroscopy of an in vitro model of human cervical neoplasia identifies graded spectral shape changes with neoplastic phenotype and a differential effect of acetic acid. Cancer epidemiology, 33(6), 463-468. http://dx.doi.org/10.1016/j.canep.2009.10.010 
Masilamani, V., Trinka, V., Al Salhi, M., Elangovan, M., Raghavan, V., Al Diab, A. R., ... Al-Nachawati, H. (2011). A new lung cancer biomarker-a preliminary report. Photomedicine and laser surgery, 29(3), 161-170. http://dx.doi.org/10.1089/pho.2009.2615

Peng, C., Gao, S., Liu, Y., Lan, X., Shen, Z., Lu, J., \& Ni, X. (2003). Investigation of LED-induced fluorescent spectra from human blood solution. Applied laser, 23(4), 232-235. 